\title{
Scaling characteristics of ULF geomagnetic fields at the Guam seismoactive area and their dynamics in relation to the earthquake
}

\author{
N. Smirnova ${ }^{1}$, M. Hayakawa ${ }^{2}$, K. Gotoh ${ }^{1,2}$, and D. Volobuev ${ }^{1}$ \\ ${ }^{1}$ St. Petersburg University, St. Petersburg 198504, Russia \\ ${ }^{2}$ The University of Electro-Communications, Chofu, Tokyo 182, Japan
}

Received: 16 May 2001 - Revised: 14 December 2001 - Accepted: 19 December 2001

\begin{abstract}
The long-term evolution of scaling (fractal) characteristics of the ULF geomagnetic fields in the seismoactive region of the Guam Island is studied in relation to the strong $(M s=8.0)$ nearby earthquake of 8 August 1993. The selected period covers 10 months before and 10 months after the earthquake. The FFT procedure, Burlaga-Klein approach and Higuchi method, have been applied to calculate the scaling exponents and fractal dimensions of the ULF time series. It is found that the spectrum of ULF emissions exhibits, on average, a power law behaviour $S(f) \propto f^{-\beta}$, which is a fingerprint of the typical fractal (self-affine) time series. The spectrum slope $\beta$ fluctuates quasi-periodically during the course of time in a range of $\beta=2.5-0.7$, which corresponds to the fractional Brownian motion with both persistent and antipersistent behaviour. An tendency is also found for the spectrum slope to decrease gradually when approaching the earthquake date. Such a tendency manifests itself at all local times, showing a gradual evolution of the structure of the ULF noise to a typical flicker noise structure in proximity to the large earthquake event. We suggest considering such a peculiarity as an earthquake precursory signature. One more effect related to the earthquake is revealed: the longest quasiperiod, which is 27 days, disappeared from the variations of the ULF emission spectrum slope during the earthquake, and it reappeared three months after the event. Physical interpretation of the peculiarities revealed has been done on the basis of the SOC (self-organized criticality) concept.
\end{abstract}

\section{Introduction}

In our recent papers by Hayakawa et al. (1999, 2000a) and Smirnova et al. (1999), a fractal analysis has been applied to ULF geomagnetic data to investigate dynamics of their scaling characteristics (spectral exponents, fractal dimensions) depending on seismic and geomagnetic conditions. It has been shown that some specific dynamics of spectral expo-

Correspondence to: N. Smirnova (nsmir@geo.phys.spbu.ru) nents (spectrum slopes) of the ULF emissions were observed in a seismoactive region of Guam Island under the variation of geomagnetic activity and in relation to the large earthquake of 8 August $1993(M=8)$. In particular, the spectrum slope, which had been calculated using the FFT method for a 1-hour interval of data in the noon and midnight sectors, is found to have a tendency to decrease when approaching the date of the earthquake. Such a specific behaviour of the spectrum slope serves as a candidate for an earthquake precursory signature. Since the effect revealed (in the case of its confirmation on new experimental materials and by other independent methods) could be important for the development of earthquake forecasting methods, it seems to be reasonable to check this effect using the more advanced methods of data analysis. In our case of the fractal analysis, the BurlagaKlein approach (Burlaga and Klein, 1986), and the Higuchi method (Higuchi, 1988, 1990) appear to be the more promising tools for calculation of scaling characteristics of time series. The efficiency of those methods has been shown on different kinds of geophysical data sets: magnetic, geo-electric, cosmic ray, and others (see, for example, Yasue et al., 1996; Telesca et al., 1999; Cervantes de la Torre et al., 1999). The purpose of this paper is to apply the fractal techniques, developed by Burlaga-Klein and Higuchi, to the Guam ULF geomagnetic data in order to identify the earthquake precursory signatures revealed by the FFT method. Also, we extend our consideration to all local time sectors to investigate the local time dependence of the peculiarities revealed.

The paper is organized as follows. In Sect. 2, we describe the experimental data chosen for analysis and present our methodology for data processing. In Sect. 3, we give the results obtained by the FFT method and discuss its applicability for processing an irregular time series. In Sect. 4, we introduce the Burlaga-Klein and Higuchi methods as the advanced methods for calculation of the time series fractal dimensions, and we test their efficiency on the simulated noise time series and apply them to the Guam data. In Sect. 5, we discuss the results obtained by different methods and outline a possibility to use scaling characteristics of the ULF geo- 


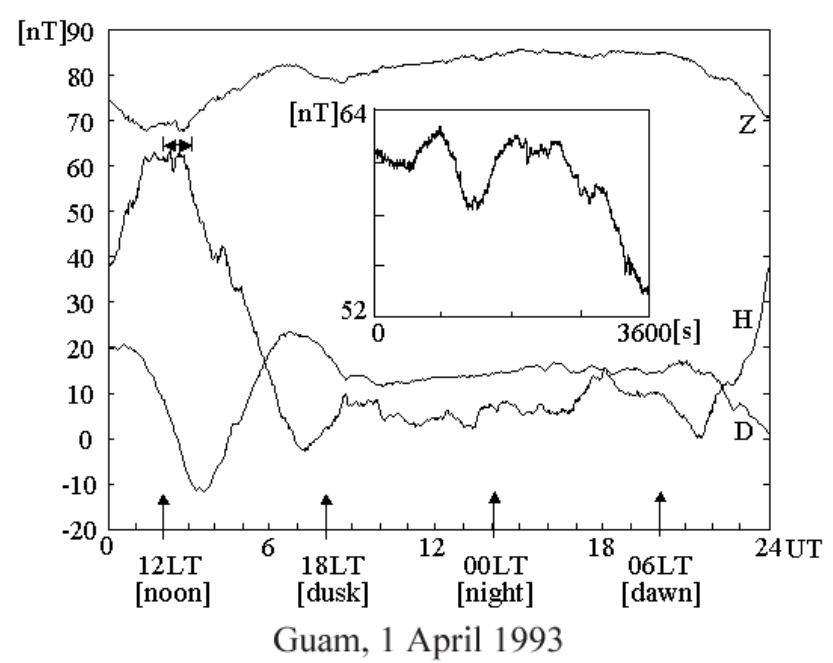

Fig. 1. An example of the daily record of geomagnetic field variations ( $H, D, Z$ components) at the Guam observatory (1 April 1993). In the insertion, one can see the original ULF record (onesecond sampling) at the 02:00-03:00 UT interval, which corresponds to the local noon sector (12:00-13:00 LT).

magnetic time series for the study of the physical processes related to the preparation stage of earthquakes.

\section{Experimental data and the analysis methodology}

Our analysis is related to the strong ( $M s=8.0)$ Guam earthquake of 8 August 1993, and its focus was located in the sea near the Guam Island $\left(\varphi=12.98^{\circ} \mathrm{N}, \lambda=144.80\right)$ at the depth of $60 \mathrm{~km}$. The raw data used for analysis are the digital records of the magnetic field components $H, D, Z$ with a one-second sampling rate, taken from the nearby magnetic observatory situated at the Guam Island $65 \mathrm{~km}$ from the epicentre. Here the regular measurements of geomagnetic field variations are maintained using a three axes ringcore type of fluxgate magnetometer, and the detailed description of the measuring system can be found in Yumoto et al. $(1992,1996)$. The chosen sampling rate allows us to investigate the properties of ULF emissions (geomagnetic pulsations) in a wide frequency range from $f=0.001 \mathrm{~Hz}$ to $f=0.3 \mathrm{~Hz}$. This frequency range is also favourable for detection of electromagnetic signals of lithospheric origin, which could be related to the earthquake preparation processes. The corresponding consideration based on the field observations and also on the estimations of the appropriate skin depths for electromagnetic waves is contained in Kopytenko et al. (1993a, b, 1994, 2001), Hayakawa et al. (1996, 1999, 2000b), and Smirnova (1999). The calculations of skin depth values show the ability of the ULF electromagnetic fields of space origin to penetrate the earthquake focal areas. The ULF part of the electromagnetic fields, which can be generated in the earthquake focus before and during the major rupture, is also able to propagate from the focus to the Earth's surface. Since the earthquake preparation processes can be connected with an alteration of the medium structures in the earthquake focal area and with the change in their elastic and electric parameters, such processes could also be manifested in the alteration of the structure of seismic and electromagnetic noise recorded near the epicentre region. Since such an alteration is expected to have a the long-term character, we give our attention to the long-term variation of scaling characteristics of the observed signals. Thus the entire investigated period covers nearly 10 months before and 10 months after the event. In our consideration, we take into account the phenomenological model proposed by Troyan et al. (1999) to describe the large-scale evolutionary SOC processes involved between two massive earthquakes.

An example of the experimental data used for the analysis is presented in Fig. 1. Here, a typical daily record of geomagnetic field variations ( $H, D, Z$ components) at Guam is shown. The original ULF record (with one-second sampling rate) can be seen in the insertion into Fig. 1. The signal is taken from 02:00-03:00 UT interval, which corresponds to local noon sector (12:00-13:00 LT). Before using the main procedure of data analysis, some selection of data has been made. First, we divide the raw data along local time intervals, thus forming for every day 24 sets of 1-hour time series, each having $N=3600$ variables ( $H, D, Z$ components). Such selection allows us to separate the local time effects from other effects that could be related to earthquake source dynamics. Scaling characteristics of the time series were calculated by using three methods of data processing: spectral analysis (FFT), the Burlaga-Klein (BK) method and the Higuchi (H) method. First, we present the results obtained by the traditional FFT (Fast Fourier Transform) method.

\section{Spectral analysis of the ULF geomagnetic data (FFT procedure)}

The observational geophysical data are usually represented by an irregular time series. To obtain a quantitative estimation of the time series irregularity, the power spectrum analysis has been conventionally used. In the case when the power spectral density follows a power law, the spectral exponent can be considered as one of the indexes for representing the irregularity of a time series. We used the FFT technique to calculate the power spectral density of the ULF signals $S(f)$ in each one-hour interval during the period October 1992 - June 1994. If $S(f)$ followed a power law behaviour $S(f) \propto f^{-\beta}$, then we calculated the spectral exponent $\beta$ from the slope of the best-fit straight line in the log-log plot of the spectrum in the frequency range $f=0.003-0.3 \mathrm{~Hz}$. An example of such a processing is given in Fig. 2. The trend was initially removed from the original signal, and the Hanning window was used for correction of the signal before applying the FFT procedure. Then we analysed the evolution of the slope $\beta$ of the spectrum in different local times, under a variety of geomagnetic conditions and on the different time distances from the earthquake. 

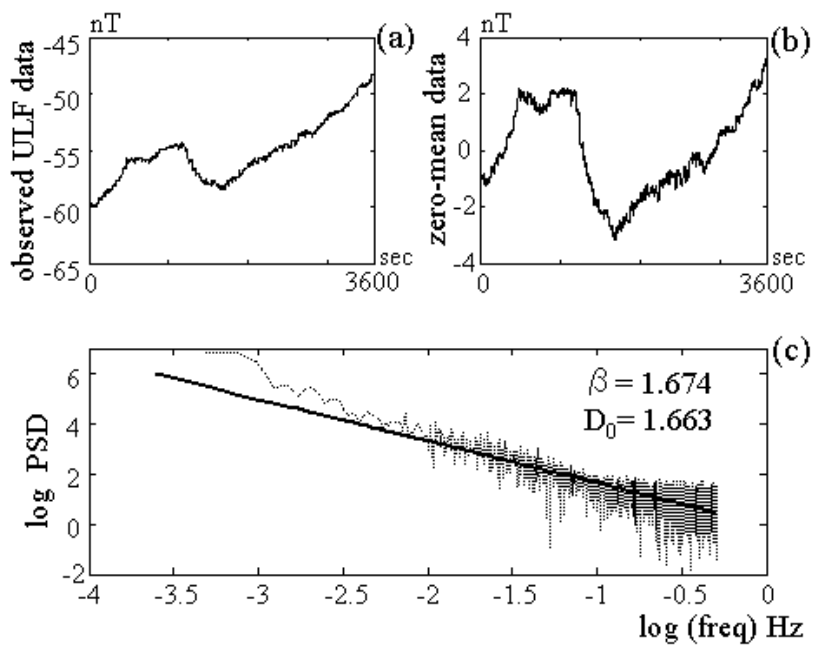

Guam, 9 April 1993

(night sector)

Fig. 2. An example of the ULF data processing by the FFT method. (a) an original signal; (b) the zero mean signal without trend; (c) the power spectrum. The best-fit line represents the spectrum slope $\beta$. The noisy fluctuations, superposed on the power law spectrum, are clearly seen.

The power law behaviour with a single $\beta$ is a characteristic feature of the statistically self-affine (fractal) time series. Statistically self-affine means that the time series $X(t)$ can be viewed geometrically as a curve, each part of which is a reduced scale image of the whole. In this context the increment function $[X(t+\tau)-X(t)] \tau^{-H u}$ has a probability distribution independent of $t$. Here, $H u$ is the Hurst exponent, which lies anywhere in the range of $0<H u<1$ (see Mandelbrot, 1977, Feder, 1988, and Turcotte, 1997). The fractal dimension $D_{0}$, which characterises the rate of the irregularity of the time series (how smooth or crinkly is the plot representing the observational data), can be calculated from the spectral exponent using the well-known Berry's equation: $D_{0}=(5-\beta) / 2$ (Berry, 1979, see also Turcotte, 1997). The dynamics of spectral exponent $\beta$ for the Guam ULF geomagnetic data is presented in Fig. 3. The $\beta$ values are calculated for the noon sector (12:00-13:00 LT) for the entire investigated period 1 October $1992-8$ June 1994. The moment of the earthquake of 8 August 1993 is marked by the arrow with symbol EQ. One can see the break in the magnetic record just after the earthquake, which lasts until the middle of September. The thin line indicates the daily values of $\beta$, and the thick line represents the 5-day running average values.

The following peculiarities can be seen from Fig. 2. Over the course of time the spectrum slope $\beta$ fluctuates quasiperiodically in a range of $\beta=2.5-0.7$. Such values of $\beta$ are typical for the fractional Brownian motion. By taking into account the Berry's equation and the relation between fractal dimension $D_{0}$ and the Hurst exponent $H u: D_{0}=2-H u$ (Hurst, 1951; Feder, 1988; Voss, 1989; Turcotte, 1997), we can estimate the range of the Hurst exponent variations and thus investigate the persistence of our time series. We can see that during the investigated period, the ULF time series at Guam are characterized by both persistent $(H u>0.5)$ and antipersistent $(H u<0.5)$ behaviour with prevalence of the antipersistent dynamics. The random Brownian walk, which corresponds to $H u=0.5(\beta=2)$, also manifests itself for a while in the time series. The next peculiarity, which can be seen from the running average plot in Fig. 2, is a quasi-periodical variation of $\beta$ with $\mathrm{T} \approx 27$ days, which corresponds to the period of the Sun's rotation. The same 27-day quasi-period is also manifested in the dynamics of the $K p$ index of the geomagnetic activity considered for the same period (see Hayakawa et al., 1999; Smirnova, 2001). This shows the influence of helio-geomagnetic conditions on the scaling characteristics of the ULF emissions. In relation to the earthquake, the most principal peculiarity, which can be seen from Fig. 2, is a gradual decrease of $\beta$ toward $\beta=1$ (flicker noise signature) when approaching the earthquake date. This indicates the appearance of the more highfrequency fluctuations in the ULF geomagnetic noise before the earthquake. The fractal dimension of the ULF time series calculated from the Berry's expression has a tendency to increase toward $D_{0}=2$ prior to the earthquake. Both the appearance of higher-frequency fluctuations and the increase in $D_{0}$ are consistent with the formation of small-scale fractal structures before the earthquake.

But there are some problems with application of the FFT method to an irregular time series. In the actual analysis of geophysical data, the power spectrum obtained by the FFT method shows noisy fluctuations superposed on the power law spectrum (see, for example, Figs. 2 and 3 in Cervantes de la Torre et al., 1999 and Fig. 4 in Telesca et al., 1999). Such fluctuations are always presented in the power spectrum of the Guam geomagnetic data (see Fig. 3c). In order to obtain a stable power law index, it is necessary to average the power spectra over a long interval where the fluctuations are statistically stationary. But in many cases it is not appropriate since the statistical characteristics of the fluctuations usually vary on the short time intervals. So the values of the spectrum slopes obtained by FFT methods may contain appreciable errors. Also, when using the FFT method, we have to remove the trend from the time series and smooth the data near the boundaries. Some part of the original information can be lost during such procedures. So we have to search for other more stable and precise methods of calculations of the spectral exponents. One example of such methods can be based on the estimation of the time series fractal dimension $D_{0}$, since this quantity is specially introduced for irregular time series. After obtaining the stable and precise value of $D_{0}$, one can estimate the spectral exponent using the Berry's expression. The advanced technique for calculation of the time series fractal dimension has been proposed by Burlaga and Klein (Burlaga and Klein, 1986), and by Higuchi (Higuchi, 1988, 1990). In the next section, we introduce those fractal methods and apply them to the Guam geomagnetic data to check the effects revealed by the FFT method. 


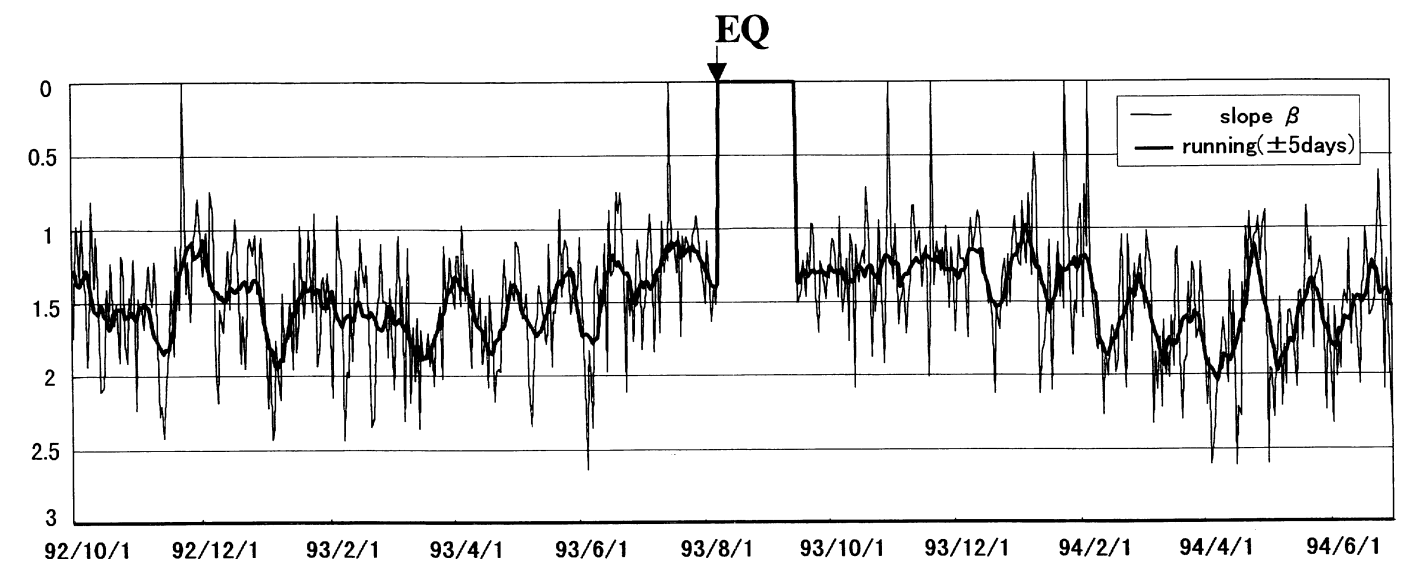

Fig. 3. The dynamics of spectral exponent $\beta$ calculated by the FFT method for the noon sector (12:00-13:00 LT) for the period 1 October $1992-8$ June 1994. The thin line indicates the daily values of $\beta$; the thick line represents the 5-day running average values. The moment of the earthquake of 8 August 1993 is marked by the arrow with symbol EQ. One can see the break in the magnetic record, which has lasted until the middle of September.

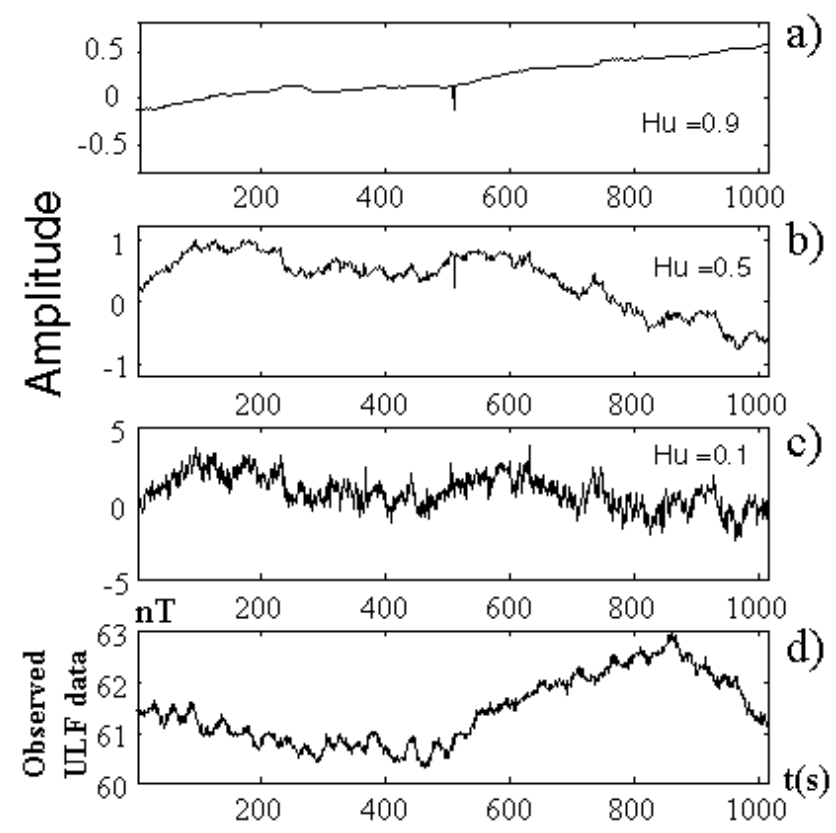

Fig. 4. Examples of simulated (a-c) and observational (d) signals examined: (a) the persistent noise with $H u=0.9$; (b) random noise $(H u=0.5)$; (c) antipersistent noise with $H u=0.1$; (d) the Guam ULF magnetic data along one-hour local noon interval (12:00-13:00 LT) of 1 April 1993.

\section{Application of the Burlaga-Klein and Higuchi methods}

To provide a stable estimation of spectral exponents, Burlaga and Klein suggested a method for calculation of stable values of the fractal dimension $D_{0}$ of geophysical time series (Burlaga and Klein, 1986). The authors used as raw data the interplanetary magnetic field variations $B(t)$. They define the length $L$ of the curve representing $B(t)$ as

$$
L_{B K}(\tau)=\sum_{k=1}^{n}\left|\bar{B}\left(t_{k}+\tau\right)-B\left(t_{k}\right)\right| / \tau
$$

where $\bar{B}\left(t_{k}\right)$ denotes the average value of $B(t)$ between $t=t_{k}$ and $t_{k}+\tau$. This length is a function of $\tau$, and for statistically self-affine curves, the length is expressed as $L_{B K}(\tau) \propto \tau^{-D_{0}}$. Using this relation, one can estimate the value of $D_{0}$ as the slope of the log-log plot of the length $L_{B K}(\tau)$ versus the time interval $\tau$. Then the power-law index $\beta$ of the power spectrum can be calculated from the Berry's expression as $\beta=5-2 D_{0}$.

The other method which gives stable values of the fractal dimension has been proposed by Higuchi (Higuchi, 1988, 1990). He modified the Burlaga-Klein method, suggesting the following procedure for calculation of the length of the curve $X(t)$. First, he constructed a new time series $X_{\tau}^{m}$ from the original time series $X(i),(i=1,2, \ldots, N)$,

$X_{\tau}^{m} ; X(m), X(m+\tau), X(m+2 \tau), \ldots$,

$X\left(m+\left[\frac{N-m}{\tau}\right] \cdot \tau\right)(m=1,2, \ldots, \tau)$,

where [ ] denotes Gauss' notation. He defines the length of curve, $X_{\tau}^{m}$, as follows:

$$
\begin{aligned}
& L_{m}(\tau)= \\
& \qquad\left(\sum_{i=1}^{\left[\frac{n-m}{\tau}\right]}|X(m+i \tau)-X(m+(i-1) \cdot \tau)|\right) \\
& \left.\frac{N-1}{\left[\frac{N-m}{\tau}\right] \cdot \tau}\right\} / \tau .
\end{aligned}
$$

The length of the curve for the time interval $\tau,\langle L(\tau)\rangle$, is defined as the average value over $m$ sets of $L_{m}(\tau)$. If $\langle L(\tau)\rangle \propto \tau^{-D_{0}}$ within the range $\tau_{\min } \leq \tau \leq \tau_{\max }$, then the curve is fractal with the dimension $D_{0}$ in this range. 
Table 1. Results of test calculations of the fractal dimensions $D_{0}$ and spectral exponents $\beta$ for simulated noise shown in Fig. 3a-c

\begin{tabular}{ccccccc}
\hline & \multicolumn{2}{c}{ noise09 } & \multicolumn{2}{c}{ noise 05 } & \multicolumn{2}{c}{ noise 01 } \\
& \multicolumn{2}{c}{$H u=0.9$} & \multicolumn{2}{c}{$H u=0.5$} & \multicolumn{2}{c}{$H u=0.1$} \\
\hline & $D_{0}=1.1$ & $\beta=2.8$ & $D_{0}=1.5$ & $\beta=2$ & $D_{0}=1.9$ & $\beta=1.2$ \\
FFT & $1.56 \pm 0.21$ & 1.88 & $1.48 \pm 0.21$ & 2.05 & $1.83 \pm 0.21$ & 1.34 \\
BK & $1.76 \pm 0.12$ & 2.48 & $1.69 \pm 0.12$ & 1.67 & $1.89 \pm 0.13$ & 1.22 \\
H & $1.18 \pm 0.04$ & 2.64 & $1.48 \pm 0.04$ & 2.04 & $1.8 \pm 0.04$ & 1.40 \\
\hline
\end{tabular}
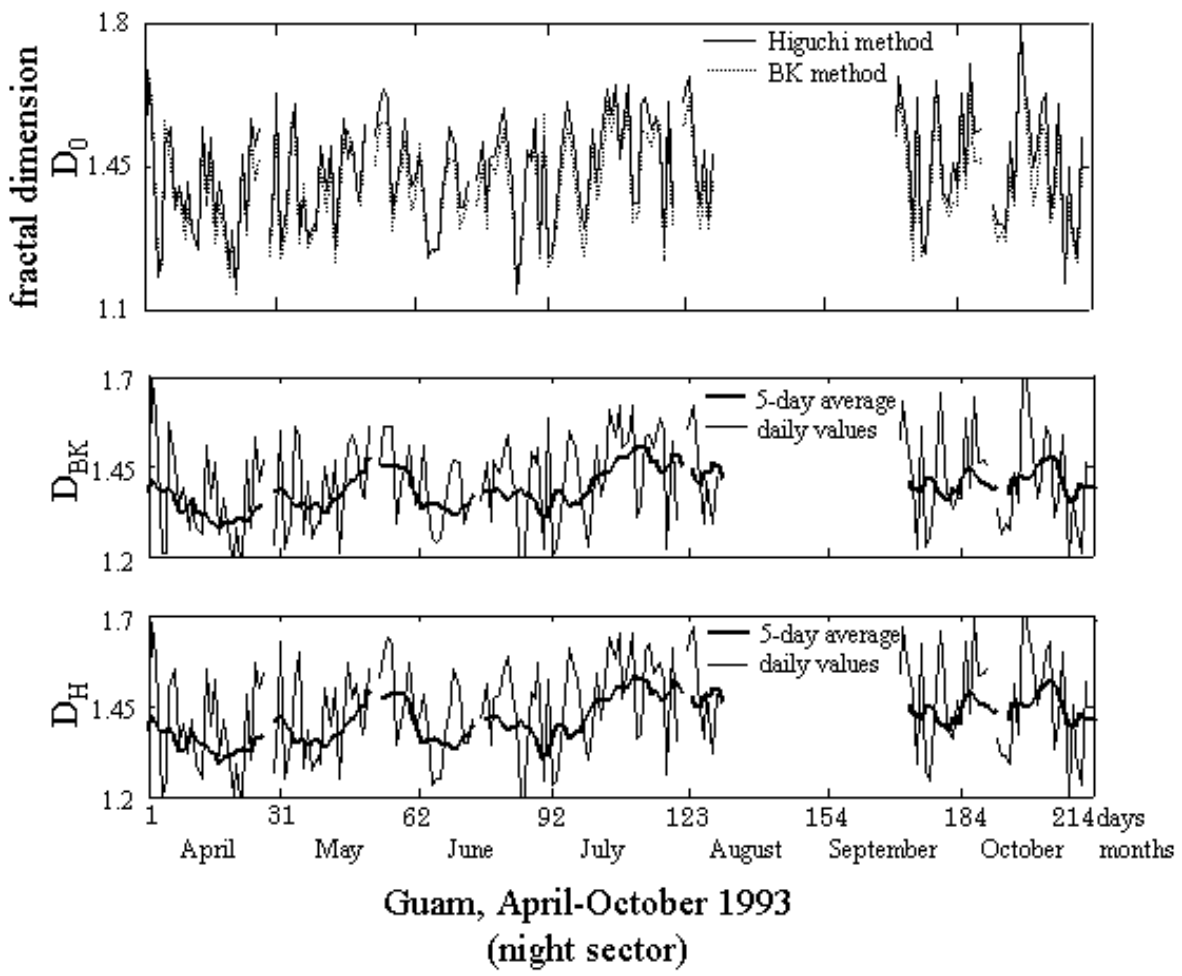

Fig. 5. Dynamics of the fractal dimension of the Guam ULF time series for the night sector calculated by the Burlaga-Klein and Higuchi methods. The curves are displayed together (upper part) and separately (lower part). In the upper part, the solid line represents the Higuchi method and the dotted line relates to the Burlaga-Klein method.

To compare the efficiency of the methods we have made test calculations of the fractal dimensions $D_{0}$ and spectral exponents $\beta$ for simulated noise with Hurst exponents $H u=$ 0.9 (persistent noise), $H u=0.5$ (random noise) and $H u=$ 0.1 (antipersistent noise). The results of data processing are presented in Table 1. The values of $D_{0}$ and $\beta$ for simulated noise are calculated from the simple relations: $D_{0}=2-H u$ and $\beta=5-2 D_{0}$ (Turcotte, 1997). The records of simulated noise for $N=1000$ can be seen in Fig. 4a-c. For comparison, we present the original signal in the lower part of Fig. 4d, i.e. the part of the ULF record shown in the insertion to Fig. 1. Let us compare first the Burlaga-Klein and Higuchi methods. It is seen from Table 1 that the Higuchi method gives the more correct estimations of $D_{0}$ and $\beta$ for persistent $(H u=0.9)$ and random $(H u=0.5)$ noise, whereas the Burlaga-Klein method gives more correct results for antipersistent $(H u=0.1)$ noise. For the noise 09 and noise 05 , frac- tal dimensions $D_{0}$ obtained by the BK method are larger than the real values. Such a tendency for the BK method to give larger values of $D_{0}$ was also mentioned by Higuchi (1988) when he explored this method. As for the FFT method, it gives the correct results for random and antipersistent noise, but its estimation for the persistent noise is less correct than the estimations by the Burlaga-Klein and Higuchi methods. As one can also see from Table 1, the largest errors correspond to the FFT method, and the smallest ones correspond to the Higuchi method.

The results of application of both Burlaga-Klein and Higuchi methods to Guam data are shown in Fig. 5. Here the dynamics of the fractal dimension $D_{B K}$ and $D_{H}$ of Guam ULF time series is presented for the night sector. The plots are displayed together in the upper part of Fig. 5 and separately in its lower part. We find nearly the similar behaviour of both curves, although the absolute values of $D_{B K}$ and $D_{H}$ 


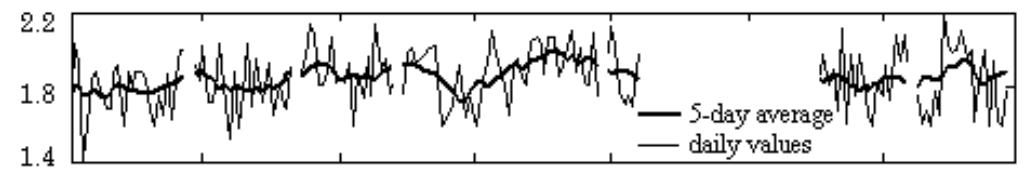

LT 0:00-1:00

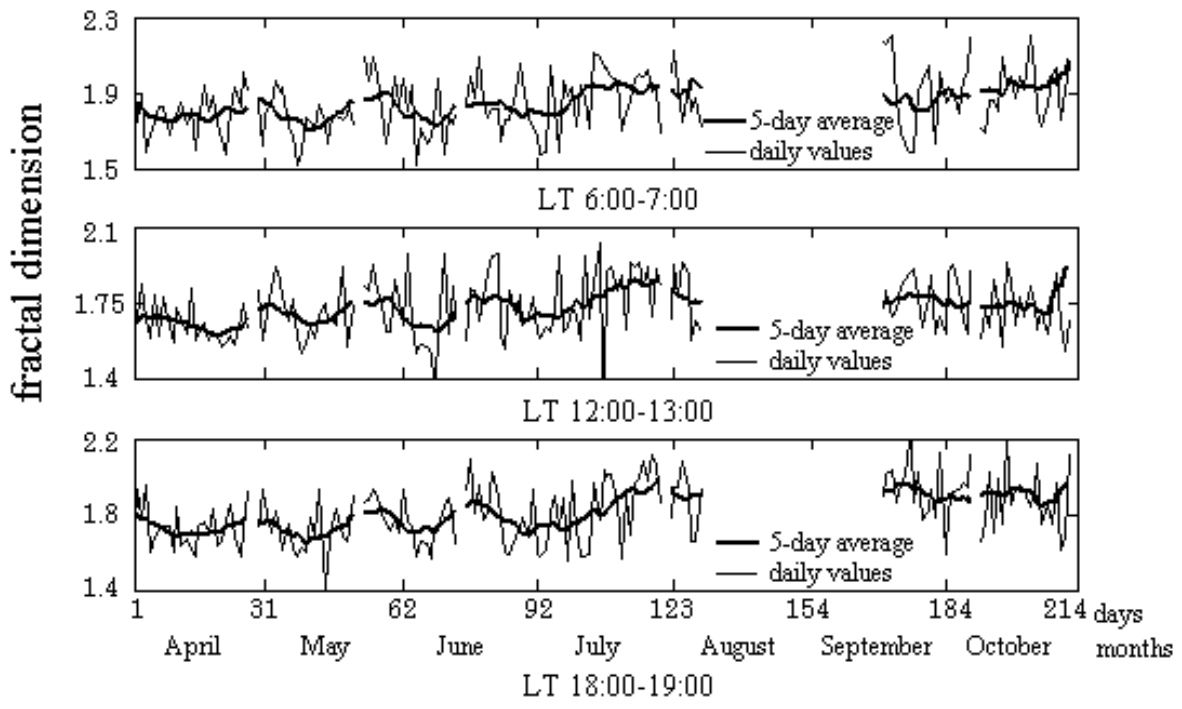

Guam, April-October 1993

Fig. 6. Dynamics of the fractal dimension for different local time sectors: night, dawn, noon, and dusk (FFT method).

differ from each other. From Fig. 5, one can also see the gradual increase in the ULF time series fractal dimension, when approaching the earthquake date, which confirms the results obtained with the FTT method.

\section{Discussion}

As it follows from Table 1, the FFT method gives results that are not so bad for random and antipersistent noise. Since the real values of $\beta$ for the Guam data occupy mainly the range $\beta=1-2$, which corresponds to random and antipersistent noise, the use of the FFT method for the Guam data seems to be correct. One can also see from Fig. 4 that the observed signal looks more like random and antipersistent noise, than the persistent one. So we can use the FTT method and extend our consideration at all local time sectors to identify the peculiarities revealed for the noon sector. The corresponding results of the calculations of the ULF time series fractal dimension $D_{0}$ are shown in Fig. 6 for different local time sectors: night (00:00-01:00 LT), dawn (06:00-07:00 LT), noon (12:00-13:00 LT), and dusk (18:00-19:00 LT). It is seen from Fig. 6 that the tendency for fractal dimension to increase before the earthquake is manifested itself at all local time intervals. The tendency seems to be more pronounced at the dusk sector. By taking into account the Berry's equation, we can conclude that the effect of the decrease in the ULF emission spectrum slope before the earthquake is really confirmed. Such specific dynamics, which start about 1-2 months before the earthquake, can be attributed to the earthquake preparation processes, when the focal area is involved in selforganized critical (SOC) dynamics. In this state, the rock medium is porous like a honeycomb, and its structure displays a more and more crinkly character. As a consequence of such fractal dynamics, the ULF electromagnetic noise, which could be generated in the process of crack dynamics (see Molchanov and Hayakawa, 1995; Vallianatos and Tzanis, 1999), is supplemented gradually by higher-frequency fluctuations. In ground-based observations, such dynamics has to be manifested as a decrease in the ULF spectrum slope and as antipersistent behaviour of the ULF time series before the earthquake. Such peculiarities are factually observed. In the SOC state, the focal system has a high risk of the massive earthquake, since any alteration at small-scales (for example, filling the small pore space by fluid) can implicate the alteration of the whole system. The corresponding explanation can be found in Hayakawa et al. $(1999,2000)$ and Troyan et al. (1999). So we can consider the revealed peculiarity (the decrease in the ULF spectrum slope prior to the earthquake) as a premonitory symptom of a catastrophic event. It this relation, it also should be mentioned that the pronounced dynamics of the polarization ratio $Z / H$ of the Guam ULF data, starting 1-2 months before the earthquake, had been revealed earlier by Hayakawa et al. (1996). Such dynamics could relate to the change in the Earth's crust conductivity, and this is also in good agreement with our consideration.

One more effect, possibly related to the earthquake, one can recognize from Fig. 3. As it is seen from the running 
average curve, the pronounced quasi-period of 27 days disappears from the variations of the spectrum slope during the earthquake, but it reappears nearly three months after the earthquake. The same peculiarity (disappearance of the pronounced 27-day period from the variation) has been revealed in dynamics of the planetary $\mathrm{Kp}$ index of geomagnetic activity (see Fig. 3 in Hayakawa et al., 1999). It means that a global alteration of the dynamics of the Earth-magnetosphere system takes place during massive earthquakes.

On the basis of our analysis we conclude that scaling characteristics of the ULF electromagnetic fields registered in a seismically active region, could give us important information on the earthquake source dynamics and allow us to gain insight into the physical mechanisms governing the earthquake preparation processes. In this paper, we do not intend to suggest any method for earthquake prediction, but we hope that the development of such research would give an appreciable contribution in solving this problem.

Acknowledgements. The work was supported by Russian Programme "Intergeophysica" and by the Grant INTAS 99-1102.

\section{References}

Berry, M. V.: Diffractals, J. Phys. A Math. Gentile, 12, 207-220, 1979.

Burlaga, L. F. and Klein, L. W.: Fractal Structure of the Interplanetary Magnetic Field, J. Geophys. Res., 91, A1, 347-350, 1986.

Cervantes de la Torre, F., Ramirez-Rojas, A., Pavia-Miller, C. G., Angulo-Brown, F., Yepez, E., and Peralta, J. A.: A comparison between spectral and fractal methods in electrotelluric time series, Revista Mexicana de Fisica, 45, 3, 298-302, 1999.

Feder, J.: Fractals, Plenum Press, New York, 283, 1988.

Hayakawa, M., Kawate, R., Molchanov, O. A., and Yumoto, K.: Results of ultra-low-frequency magnetic field measurements during the Guam earthquake of 8 August 1993, Geophys. Res. Lett., 23, 241-244, 1996.

Hayakawa, M., Ito, T., and Smirnova, N.: Fractal analysis of ULF geomagnetic data associated with the Guam earthquake on $8 \mathrm{Au}-$ gust 1993, Geophys. Res. Lett., 26, 18, 2797-2800, 1999.

Hayakawa, M., Ito, T., Hattori, K., and Yumoto, K.: ULF electromagnetic precursors for an earthquake at Biak, Indonesia on 17 February 1996, Geophys. Res. Lett., 27, 1531-1534, 2000 a.

Hayakawa, M., Kopytenko, Yu. A., Smirnova, N. A., Troyan, V. N., and Peterson, Th.: Monitoring ULF Magnetic Disturbances and Schemes for Recognizing Earthquake Precursors, Phys. Chem. Earth (A), 25, 3, 263-269, 2000 b.

Higuchi, T.: Approach to an Irregular Time Series on the Basis of Fractal Theory, Physica D 31, 277-283, 1988.

Higuchi, T.: Relationship between the Fractal Dimension and the Power-low Index for a Time Series: a Numerical Investigation, Physica D 46, 254-264, 1990.

Hurst, H. E.: Long-term storage capacity of reservoirs, Trans, Am. Soc. Civ. Eng., 116, 770-808, 1951.

Kaufman, A. A. and Keller, G. V.: : The Magnetotelluric Sounding Method, 1993.

Kopytenko, Yu. A., Matiashvili, T. G., Voronov, P. M., Kopytenko, E. A., and Molchanov, O. A.: Detection of Ultra-LowFrequency Emissions Connected with the Spitak Earthquake and its Aftershock Activity, Based on Geomagnetic Pulsations Data at Dusheti and Vardzia Observatories, Phys. Earth Planet. Inter., 77, 850-95, 1993a.

Kopytenko, Yu. A., Voronov, P. M., Kopytenko, E. A., Matiashvili, T. G., Fraser-Smith, A. C., and Bernardi, A.: Results of magnetic field measurements near the epicenters of the Spitak $(M=6.9)$ and Loma Prieta $(M=7.1)$ Earthquakes: comparative analysis, Russian Airborne Geophysics and Remote Sensing, Proc. SPIE 2111, (Ed) Harthil, N., 317-329, 1993b.

Kopytenko, Yu. A., Matiashvili, T. G., Voronov, P. M., and Kopytenko E. A.: Observation of Electromagnetic Ultra-LowFrequency Lithspheric Emissions (ULE) in the Caucasian Seismicallly Active Area and Their Connection with the Earthquakes, in: Electromagnetic Phenomena Related to Earthquake Prediction, (Eds) Hayakawa, M. and M. Fujinawa, M., Terra Sci. Pub. Co., Tokyo, 175-180, 1994.

Kopytenko, Yu., Ismagilov, V., Hayakawa, M., Smirnova, S., Troyan, V., and Peterson, Th.: Investigation of the ULF electromagnetic phenomena related to earthquakes: contemporary achievements and the perspectives, Annali di Geofisica, 44, 2, 325-334, 2001.

Mandelbrot, B. B.: Fractals: Form, Chance, Dimension, Freeman, San Francisco, Molchanov, O.A., and M Hayakawa (1995): Generation of ULF electromagnetic emissions by microfracturing, Geophys. Res. Lett., 22, 3091-3094, 1997.

Molchanov, O. A., and Hayakawa, M.: Generation of ULF electromagnetic emissions by microfracturing, Geophys. Res. Lett., 22, 3091-3094, 1995.

Smirnova, N. A.: The peculiarities of Ground-Observed Geomagnetic Pulsations as the Background for Detection of ULF Emissions of Seismic Origin, in: Atmospheric and Ionospheric electromagnetic phenomena associated with Earthquakes, (Ed) Hayakawa, M., Terra Sci. Publ. Co., Tokyo, 215-232, 1999.

Smirnova, N. A.: Characteristics of the ground observed ULF emissions and their dynamics depending on solar wind parameters and geomagnetic conditions, in: Noise in Physical Systems and 1/f Fluctuations (Proc. 16th Int. Conf. ICNF'2001, Florida, USA, 22-25 October), (Ed) Bosman, J., World Scientific, 805-808, 2001.

Smirnova, N. A., Hayakawa, M., and Ito, T.: Structure of the ULF geomagnetic noise in a seismoactive zone and its relation to the earthquake, in: Noise in Physical Systems and 1/f Fluctuations (Proc. 15th Int. Conf. ICNF'99, Hong Kong, 23-26 August), (Ed) Surya, Ch., World Scientific, 471-474, 1999.

Telesca, L., Cuomo, V., Lapenna, V., Macchiato, M., and Serio, C.: Detecting stochastic behaviour and scaling laws in time series of geomagnetic daily means, Pure Appl. Geophys., 156, 487-501, 1999.

Troyan, V. N., Smirnova, N. A., Kopytenko, Yu. A., Peterson, Th., and Hayakawa, M.: Development of a Complex Approach for Searching and Investigation of Electromagnetic Precursors of Earthquakes: Organization of Experiments and Analysis procedures, in: Atmospheric and Ionospheric electromagnetic phenomena associated with Earthquakes, (Ed) Hayakawa, M., Terra Sci. Publ. Co., Tokyo, 147-171, 1999.

Turcotte, D. L.: Fractals and Chaos in Geology and Geophysics, Cambridge University Press, Second edition, 397, 1997.

Vallianatos, F. and Tzanis, A.: A model for the generation of precursory electric and magnetic fields associated with the deformation rate of the earthquake focus, in: Atmospheric and Ionospheric electromagnetic phenomena associated with Earthquakes, (Ed) Hayakawa, M., Terra Sci. Publ. Co., Tokyo, 287-306, 1999.

Voss, R. F.: Random fractals: self-affinity in noise, music, moun- 
tains and clouds, Physica D, 38, 362-371, 1989.

Yasue, S., Munakata, K., Kato, M., and Mori, S.: Calculation of the Power-law Index for a Time Series by means of its Fractal Dimension, Fractals, 4, 3, 265-271, 1996.

Yumoto, K., Tanaka, Y., Oguti, T., Shiokawa, K., Yoshimura, U., Isono, A., Fraser, B. J., Menk, F. W., Lynn, J. W., Seto, M., and $210^{\circ} \mathrm{MM}$ magnetic observation group: Globally coordi- nated magnetic observations along $210^{\circ}$ magnetic meridian during STEP period: 1. Preliminary results of low-latitude Pc3's, J. Geomag. Geoelectr., 44, 261-276, 1992.

Yumoto, K. and $210^{\circ} \mathrm{MM}$ magnetic Observation Group: The STEP $210^{\circ}$ magnetic meridian network project, J. Geomag. Geoelectr., 48, 1297-1309, 1996. 